\title{
The thermopower as a fingerprint of the Kondo breakdown quantum critical point
}

\author{
K.-S. Kim ${ }^{1,3}$ and C. Pépin ${ }^{2,4}$ \\ ${ }^{1}$ Asia Pacific Center for Theoretical Physics, Hogil Kim Memorial building 5th floor, \\ POSTECH, Hyoja-dong, Namgu, Pohang 790-784, Korea \\ ${ }^{2}$ Institut de Physique Théorique, CEA, IPhT, CNRS, URA 2306, F-91191 Gif-sur-Yvette, France \\ ${ }^{3}$ Department of Physics, Pohang University of Science and Technology, Pohang, Gyeongbuk 790-784, Korea \\ ${ }^{4}$ International Institute of Physics, Universidade Federal do Rio Grande do Norte, 59078-400 Natal-RN, Brazil
}

(Dated: 29 mai 2022)

\begin{abstract}
We propose that the thermoelectric power distinguishes two competing scenarios for quantum phase transitions in heavy fermions : the spin-density-wave (SDW) theory and breakdown of the Kondo effect. In the Kondo breakdown scenario, the Seebeck coefficient turns out to collapse from the temperature scale $E^{*}$, associated with quantum fluctuations of the Fermi surface reconfiguration. This feature differs radically from the physics of the SDW theory, where no reconstruction of the Fermi surface occurs, and can be considered as the hallmark of the Kondo breakdown theory. We test these ideas, upon experimental results for $\mathrm{YbRh}_{2} \mathrm{Si}_{2}$.

PACS numbers: 71.27.+a, 72.15.Qm, 75.20.Hr, 75.30.Mb
\end{abstract}

A quantum transition from a light metal to a heavy Fermi liquid is believed to occur in a class of heavy fermion (HF) compounds as a result of the competition between Rudderman-Kittel-Kasuya-Yoshida interactions and the formation and condensation of Kondo singlets [1]. The Kondo breakdown theory [2-4] offers a minimal model describing this transition. It differs from the spin-density-wave (SDW) theory [5, 6] in respect that the whole heavy Fermi surface is destabilized at the quantum critical point (QCP) and a reconstruction of the Fermi surface is inevitable, sometimes identified with an orbital selective Mott transition. As a result, the Kondo breakdown QCP is described by critical fluctuations with the dynamical exponent $z=3$, associated with breakdown of the Kondo effect [3, 4], while the SDW QCP is explained within $z=2$ related with antiferromagnetic spin fluctuations [5, 6].

Until now, the thermopower has been studied mainly in the heavy Fermi liquid phase, with a special focus on its sign and its ratio with the Sommerfeld coefficient. In heavy fermions, the sign of the thermopower is determined by the position of the Kondo resonance with respect to the Fermi surface. The strong mass renormalization is proven not to affect the quasi-universal ratio to the Sommerfeld coefficient, which remains almost the same as in conventional metals [7, 8]. Little is known about the Seebeck coefficient close to the QCP. Preliminary studies for $\mathrm{CeCu}_{6-x} \mathrm{Au}_{x}$ [9] and $\mathrm{Ce}\left(\mathrm{Ni}_{1-x} \mathrm{Pd}_{x}\right)_{2} \mathrm{Ge}_{2}$ [10] show that the presence of a QCP modifies low temperature dependence of the Seebeck coefficient. Two recent studies under magnetic fields show some striking similarity between thermoelectric effects in $\mathrm{CeCoIn}_{5}$ [11] and $\mathrm{URu}_{2} \mathrm{Si}_{2}$ [12]. In particular, both systems exhibit a pronounced anisotropy in their thermoelectric response. Lastly, a recent experiment on $\mathrm{YbRh}_{2} \mathrm{Si}_{2}$ under weak magnetic fields shows some drastic variations of the magnitude of the Seebeck coefficient in both sides of the QCP [13]. Even fewer theoretical studies are available 7, 14, 15]. In the case of the
SDW the authors of Ref. [16] argued that the Seebeck coefficient divided by temperature has the same variation in temperature as the Sommerfeld coefficient and the quasi-universal ratio is preserved at the SDW QCP.

In this study we show that the thermoelectric power can be regarded as one of the hallmarks for the Kondo breakdown quantum criticality, providing a careful fit to the recent experimental observations on $\mathrm{YbRh}_{2} \mathrm{Si}_{2}$ [13]. Precisely, the abrupt collapse from the temperature identified with $E^{*}$ in the Kondo breakdown scenario [3, 4] turns out to be the unique prediction from the Kondo breakdown theory beyond the SDW [5, $\underline{6}$ ] and local quantum critical [17] scenarios.

We start from the $\mathrm{U}(1)$ slave-boson representation of the Anderson lattice model in the large- $U$ limit

$$
\begin{aligned}
& L_{A L M}=\sum_{i} c_{i \sigma}^{\dagger}\left(\partial_{\tau}-\mu\right) c_{i \sigma}-t \sum_{\langle i j\rangle}\left(c_{i \sigma}^{\dagger} c_{j \sigma}+H . c .\right) \\
& +V \sum_{i}\left(b_{i} f_{i \sigma}^{\dagger} c_{i \sigma}+H . c .\right)+\sum_{i} b_{i}^{\dagger} \partial_{\tau} b_{i} \\
& +\sum_{i} f_{i \sigma}^{\dagger}\left(\partial_{\tau}+\epsilon_{f}\right) f_{i \sigma}+J \sum_{\langle i j\rangle}\left(f_{i \sigma}^{\dagger} \chi_{i j} f_{j \sigma}+H . c .\right) \\
& +i \sum_{i} \lambda_{i}\left(b_{i}^{\dagger} b_{i}+f_{i \sigma}^{\dagger} f_{i \sigma}-1\right)+N J \sum_{\langle i j\rangle}\left|\chi_{i j}\right|^{2} .
\end{aligned}
$$

Here, $c_{i \sigma}$ and $d_{i \sigma}=b_{i}^{\dagger} f_{i \sigma}$ are conduction electron with a chemical potential $\mu$ and localized electron with an energy level $\epsilon_{f}$, where $b_{i}$ and $f_{i \sigma}$ are called holon and spinon, representing hybridization and spin fluctuations, respectively. The spin-exchange term for the localized orbital is introduced as a competitor of the hybridization term, and decomposed via exchange hopping processes of spinons, where $\chi_{i j}$ is a hopping parameter for the decomposition. $\lambda_{i}$ is a Lagrange multiplier field to impose the single occupancy constraint $b_{i}^{\dagger} b_{i}+f_{i \sigma}^{\dagger} f_{i \sigma}=N / 2$, where $N$ is the number of fermion flavors with $\sigma=1, \ldots, N$.

Performing the saddle-point approximation of $b_{i} \rightarrow b$, 
$\chi_{i j} \rightarrow \chi$, and $i \lambda_{i} \rightarrow \lambda$, one finds an orbital selective Mott transition as Kondo breakdown at $J \approx T_{K}$. For $\left\langle b_{i}\right\rangle=0$, a spin-liquid Mott insulator arises with a small area of the Fermi surface in $J>T_{K}$ while for $\left\langle b_{i}\right\rangle \neq 0$ a heavy Fermi liquid obtains with a large Fermi surface in $T_{K}>J$ [2-4]. Here, $T_{K}=D \exp \left(\frac{\epsilon_{f}}{N \rho_{c} V^{2}}\right)$ is the singleion Kondo temperature, where $\rho_{c} \approx(2 D)^{-1}$ is the density of states for conduction electrons with the half bandwidth $D$. Reconstruction of the Fermi surface occurs at $J \simeq T_{K}$.

The fluctuation-corrections are taken into account in the Eliashberg framework [3, 4]. The main physics is that the Kondo breakdown QCP is multi-scale. The dynamics of the hybridization fluctuations is described by $z=3$ critical theory due to Landau damping of electron-spinon polarization above an intrinsic energy scale $E^{*}$, while by $z=2$ dilute Bose gas model below $E^{*}$. The energy scale $E^{*}$ originates from the mismatch of the Fermi surfaces of the conduction electrons and spinons, one of the central aspects in the Kondo breakdown scenario. Physically, one may understand that quantum fluctuations of the Fermi surface reconfiguration start to be frozen at $T \approx E^{*}$, thus the conduction electron's Fermi surface dynamically decouples from the spinon's one below $E^{*}$. We show that the Seebeck coefficient collapses at $E^{*}$, associated with the Fermi surface reconstruction.

The thermoelectric power can be deduced from the following transport equations

$$
\begin{aligned}
J_{e l}^{c} & =\sigma_{c}\left(E-\nabla \mu_{c}\right)-p_{c} \nabla T, \\
J_{t h}^{c} & =T p_{c}\left(E-\nabla \mu_{c}\right)-\kappa_{c} \nabla T, \\
J_{e l}^{f} & =\sigma_{f}\left(\epsilon-\nabla \mu_{f}\right)-T p_{f} \nabla T, \\
J_{t h}^{f} & =T p_{f}\left(\epsilon-\nabla \mu_{f}\right)-\kappa_{f} \nabla T, \\
J_{e l}^{b} & =\sigma_{b}\left(-E+\epsilon-\nabla \mu_{b}\right)-p_{b} \nabla T, \\
J_{t h}^{b} & =T p_{b}\left(-E+\epsilon-\nabla \mu_{b}\right)-\kappa_{b} \nabla T,
\end{aligned}
$$

defining three transport coefficients of $\sigma, p$, and $\kappa$, where three species of conduction electrons, holons, and spinons are taken into account for each response function, denoted by $c, b$, and $f$, respectively. $J_{e l(t h)}$ is the electric (thermal) current, and $E, \epsilon, \mu, T$ are an external electric field, internal one, chemical potential and temperature, respectively. The internal electric field $\epsilon$ emulates the "back-flow" condition $J_{e l}^{f}+J_{e l}^{b}=0$ [18], central to transport in gauge theories. Taking $\mu_{c}=\mu_{f}-\mu_{b}$ with an open-circuit boundary condition $J_{e l}^{c}-J_{e l}^{b}=0$, we find the total thermopower conductivity $p_{t}(T)$ and electrical one $\sigma_{t}(T)$

$$
\begin{aligned}
& p_{t}(T)=p_{c}(T)+\frac{\sigma_{b}(T) p_{f}(T)-\sigma_{f}(T) p_{b}(T)}{\sigma_{b}(T)+\sigma_{f}(T)} \\
& \sigma_{t}(T)=\sigma_{c}(T)+\frac{\sigma_{b}(T) \sigma_{f}(T)}{\sigma_{b}(T)+\sigma_{f}(T)}
\end{aligned}
$$

Then, the physical Seebeck coefficient is given by $S_{t}(T) \equiv$ $-p_{t}(T) / \sigma_{t}(T)$.
Around the QCP, the Seebeck coefficient can be simplified as follows

$S_{H F}(T) \approx-\frac{p_{c}(T)+p_{f}(T)}{\sigma_{c}(T)+\sigma_{f}(T)}, \quad S_{Q C, S L}(T) \approx-\frac{p_{c}(T)}{\sigma_{c}(T)},(6)$

based on the fact that $\sigma_{f}(T) \ll \sigma_{b}(T) \rightarrow \infty$ in the HF phase and $\sigma_{f}(T) \gg \sigma_{b}(T) \rightarrow 0$ in both the quantum critical regime and spin liquid state.

Evaluating the thermopower conductivity in the oneloop level

$$
\begin{aligned}
& p_{c(f)}(T)=N \rho_{c(f)} v_{F}^{c(f) 2} \int_{-\infty}^{\infty} d \omega\left(-\frac{\partial f(\omega)}{\partial \omega}\right)\left(\frac{\omega}{T}\right) \\
& \int_{-\infty}^{\infty} d k\left[\Im G_{c(f)}(k, \omega+i \delta)\right]^{2},
\end{aligned}
$$

where $G_{c(f)}(k, \omega+i \delta)$ is the full Green's function in the Eliashberg approximation and $v_{F}^{c(f)}$ is the Fermi velocity of conduction electrons (spinons), we find

$$
\begin{aligned}
& p_{c(f)}(T) \approx-\frac{\pi^{2}}{3} \frac{2}{v_{F}^{c(f) 2} Z_{c(f)}^{3}\left(k_{F}^{c(f)}\right)}\left(\frac{\partial Z_{c(f)}(k)}{\partial k}\right)_{k_{F}^{c(f)}} \\
& \times \frac{T}{Z_{c(f)}(T)} \sigma_{c(f)}(T) \\
& \text { where } Z_{c(f)}^{-1}(T)=1-\left.\frac{\partial}{\partial \omega} \Re \Sigma_{c(f)}\left(k_{F}^{c(f)}, \omega\right)\right|_{\omega=T}, \\
& Z_{c(f)}(k)=1+\frac{1}{v_{F}^{c(f)}} \frac{\partial}{\partial k} \Re \Sigma_{c(f)}\left(k, E_{k}^{c(f)}\right)
\end{aligned}
$$

are wave-function and dispersion renormalization [19], respectively, and $\Re \Sigma_{c(f)}\left(k_{F}^{c(f)}, \omega\right)$ is the real part of the electron (spinon) self-energy. This expression is basically the same as the standard representation [7, 14, 15], where the derivative of the density of states with respect to frequency is replaced with that of the dispersion renormalization function with respect to momentum. Thus, the sign of the Seebeck coefficient is given to be negative when the Kondo resonance lies below the Fermi energy, exactly the $\mathrm{Yb}$ case.

An important quantity is the quasiparticle weight $Z_{c(f)}(\omega)$, resulting from the linear dependence of frequency in the thermopower expression. A singular logarithmic temperature dependence is revealed in the quantum critical regime, typical of the $z=3$ quantum criticality in three dimensions beyond the SDW theory [16].

The self-energy correction can be found within the Eliashberg theory, where quantum corrections are selfconsistently introduced in the one-loop level but vertex corrections are neglected [3, 4]. The dispersion renormalization function is obtained from the renormalized band. In the HF phase the spinon and conduction electron bands are hybridized. Resorting to these two renormalization functions, we can provide a fit for the recent experimental data for $\mathrm{YbRh}_{2} \mathrm{Si}_{2}$ [13]. The main feature 
of the experimental results is that the Seebeck coefficient divided by temperature is found to increase logarithmically in temperature above a certain energy scale $T_{M}$ and drops down abruptly in both the quantum critical regime and antiferromagnetic phase while it saturates to a constant in the heavy Fermi liquid. The Kondo breakdown QCP scenario reproduces this main feature almost exactly as shown in Fig. 1. The $\log T$ divergence is explained by the $z=3$ quantum critical regime of the theory. In particular, the collapse at $T_{M}$ is attributed to suppression of quantum fluctuations of Fermi surfaces at $E^{*}$, thus $T_{M}=E^{*}$ in the Kondo breakdown theory, where localized f-electrons cannot participate in carrying entropy due to the Fermi surface decoupling. In this regime the question whether $S / T$ changes sign at very low temperatures is still experimentally under scrutiny. In the Kondo breakdown theory, the negative sign of the Seebeck coefficient is attributed to the presence of the fluctuating hybridization between the f-spinons and the conduction electrons, yielding to the formation of the Kondo resonance below the Fermi level. Below $E^{*}$ however, the spinon Fermi surface decouples from the conduction electron one, and $S / T$ saturates to the value determined solely by the light conduction electrons. In the heavy Fermi liquid the Kondo breakdown theory shows the saturation associated with Fermi liquid physics, but without changing sign.

To understand better the quantum critical region, we approximate the self-energy and obtain the typical $z=3$ form analytically in three dimensions,

$\Sigma_{c(f)}\left(k_{F}^{c(f)}, \omega>E^{*}\right)=-\frac{m_{b} V^{2}}{6 v_{F}^{f(c)}} \omega \ln \left(\frac{\alpha D}{\omega}\right)+i \frac{m_{b} V^{2}}{12 \pi v_{F}^{f(c)}}|\omega|$,

where $D$ is the half-bandwidth and $m_{b}=\left(2 N V^{2} \rho_{c}\right)^{-1}$ is the band mass of holons. Then, the two renormalization functions are given by

$$
\begin{aligned}
& Z_{c}^{Q C-1}(T)=1-\frac{m_{b} V^{2}}{6 v_{F}^{f}}+\frac{m_{b} V^{2}}{6 v_{F}^{f}} \ln \left(\frac{\alpha D}{T}\right) \\
& \left(\frac{\partial Z_{c}^{Q C}(k)}{\partial k}\right)_{k_{F}^{c}}=\left(\frac{\partial Z_{c}^{H F}(k ; b \rightarrow 0)}{\partial k}\right)_{k_{F}^{c}} \approx-\frac{1}{4 \alpha q^{*}}(8)
\end{aligned}
$$

displaying the $\log T$-dependence and negative sign due to the f-resonance below the Fermi energy.

Lastly, we show that in the $\mathrm{HF}$ regime $\left(V>V_{c}\right)$, the saturation value of the Seebeck coefficient divided by the temperature $\alpha \equiv-\frac{S_{H F}(V)}{T}$ is proportional to $B-B^{*}$, where $B^{*}$ is slightly displaced from the critical field $B_{c}$ of the antiferromagnetic $\mathrm{QCP}$ in $\mathrm{YbRh}_{2} \mathrm{Si}_{2}$. Resorting to the condensation amplitude $b^{2} \approx \mathcal{C} \frac{\ln \alpha^{-1}}{(1-\alpha)}\left(\frac{-\epsilon_{f}}{D}\right)\left(J_{K}-\right.$ $\left.J_{K}^{c}\right)$, where $\mathcal{C}$ is a positive numerical constant from the mean-field analysis, we obtain

$$
\alpha \propto \mathcal{C} \frac{-\epsilon_{f}}{v_{F}^{f 2} q^{* 2}} \frac{\ln \alpha^{-1}}{(1-\alpha)} J_{K}^{c}\left(J_{K}-J_{K}^{R}\right)
$$
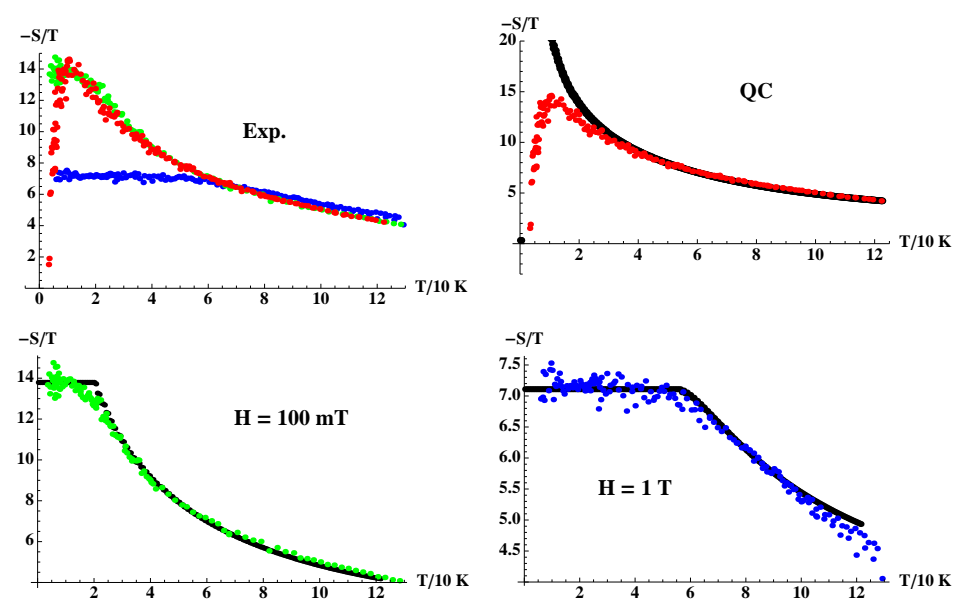

Figure 1: (Color online) Fitting to the experimental data of Ref. [13] based on the analytic expression of Eq. (7). The values of the parameters are the half bandwidth of the conduction electrons $D=990 K$ [20], of the spinons $D_{f}=1 K$, and $E^{*}=40 \mathrm{mK}$. The upper left quadrant represents the experimental data. The upper right quadrant fits the QC regime. An overall adjusting coefficient $A=0.33$ has been used. The holon mass is $m=6.0 \times 10^{-4} \mathrm{~K}$. The lower left quadrant corresponds to a field of $100 \mathrm{mT}$. A coefficient $A=0.3$ has been used with holon mass $m=5.5 \times 10^{-4} K$. The lower right quadrant corresponds to a field of $1 T$. A coefficient $A=0.0135$ has been used with holon mass $m=-4.1 \times 10^{-4} K$. The $\log T$ behavior of the $z=3 \mathrm{QC}$ regime is correctly reproduced while the scale $E^{*}$ corresponding to the reconfiguration of the Fermi surface is found to be a bit small.

where $J_{K}^{R} \equiv J_{K}^{c}-\frac{v_{F}^{f 2} q^{* 2}}{-\epsilon_{f}} \frac{(1-\alpha)}{\ln \alpha^{-1}} \frac{1}{\mathcal{C} J_{K}^{c}} \approx J_{K}^{c}$. Assuming $J_{K} \propto B$ valid near the QCP, we conclude $\alpha \propto B-B^{*}$, consistent with the experimental data of Ref. [13].

The weak point of the Kondo breakdown theory is the treatment of the anti-ferromagnetism. However, the thermoelectric power is insensitive against onset of antiferromagnetism as far as $E^{*}$ is larger than $T_{N}$, Néel temperature. Since localized f-electrons cannot carry entropy when they are decoupled from conduction electrons in the quantum level, the collapse should occur from $E^{*}$ above $T_{N}$. Actually, this is observed in $\mathrm{YbRh}_{2} \mathrm{Si}_{2}$, where $E^{*} \approx 100 \mathrm{mK}$ and $T_{N} \approx 70 \mathrm{mK}$ as the maximum Néel temperature [13]. This collapse behavior is believed to survive beyond our approximation.

This feature differs from the SDW scenario completely, well discussed in Ref. [21]. Because quantum fluctuations associated with the Fermi surface reconfiguration do not exist in the SDW theory, the Seebeck coefficient should saturate to a constant value below both the Neel temperature and $\mathrm{HF}$ coherence one. It should display a symmetric configuration for $B<B_{c}$ and $B>B_{c}$.

Another important result of the Kondo breakdown theory applied to $\mathrm{YbRh}_{2} \mathrm{Si}_{2}$ is that the sign of the Seebeck coefficient becomes positive in the low temperature limit, changing from that in the HF phase [13. Since the spinon band is decoupled from the conduction band, 
the Kondo resonance disappears even in the dynamical level and contributions from only conduction electrons give rise to a small positive constant, reflecting the physics of the normal Fermi liquid. This feature is difficult to interpret within the SDW framework without the Fermi surface reconstruction.

One may ask why the Seebeck coefficient is sensitive to $E^{*}$ while other quantities such as the specific heat coefficient 22] and thermal conductivity [23] do not show such serious dependence. The main difference between the thermoelectric power and others is that the thermodynamic and thermal transport coefficients can measure contributions of neutral spinon excitations while the Seebeck coefficient does not. This is the reason why only the thermopower is sensitive on the Fermi surface reconstruction, argued to be a fingerprint for the Kondo breakdown QCP.

Another important measurement is the Hall coefficient which also reveals an interesting energy scale, referred as $T^{*}$ in the heavy fermion side, below which the Hall coefficient $R_{H}$ shows an abrupt decrease. This abrupt change has been attributed to the proximity to a Kondo breakdown QCP [24, 25], whereas other approaches have stressed the complexity of this measurement and its sensitivity to minus fluctuations of the f-electron chemical potential [26]. Since the Hall coefficient measures the Fermi surface curvature, thus sensitive to the static formation of the heavy fermion band, the Hall number shows its characteristic only in the heavy fermion side while it does not in the localized side. On the other hand, the thermoelectric power measures fluctuations of Fermi surfaces, showing an interesting signature in the antiferromagnetic side. We refer interested readers to our future publication for a thorough study of the Hall effect within the Kondo breakdown theory.

In this paper, we argue that the thermoelectric power is an important measurement for quantum fluctuations of the Fermi surface reconfiguration, which enables us to discriminate the Kondo breakdown theory from the SDW scenario. It was demonstrated to collapse at the energy scale associated with Fermi surface fluctuations, identified with $E^{*}$ in the Kondo breakdown theory. We show here, that a careful fitting of the data can be obtained within the Kondo breakdown theory for an energy scale of the order of $40 \mathrm{mK}$, a bit small compared to the experimental value. The $z=3$ quantum criticality of Fermi surface fluctuations gives rise to the singular $\log T$ behavior in the quantum critical regime, consistent with the experimental data on $\mathrm{YbRh}_{2} \mathrm{Si}_{2}$.

The local quantum critical scenario [17] does not have the characteristic energy scale $E^{*}$ of the Kondo breakdown theory. There, the mechanism for the abrupt collapse of the Seebeck coefficient is not yet as clear as within the Kondo breakdown theory. It would be very interesting to see how the two theories of Kondo breakdown compare with respect to the fitting of the experimental data.

We thank I. Paul for very helpful discussions and S. Hartmann, N.Oeschler and F.Steglich for kindly providing their experimental data to us. K.-S. Kim was supported by the National Research Foundation of Korea (NRF) grant funded by the Korea government (MEST) (No. 2010-0074542). CP acknowledges ICAM travel fellowship and the Aspen Center for Physics where the idea of this work took form.

[1] P. Gegenwart, Q. Si, and F. Steglich, Nature Physics 4, 186 (2008) ; H. v. Lohneysen, A. Rosch, M. Vojta, and P. Wolfle, Rev. Mod. Phys. 79, 1015 (2007).

[2] T. Senthil, S. Sachdev, and M. Vojta, Phys. Rev. Lett. 90, 216403 (2003) ; T. Senthil, M. Vojta, and S. Sachdev, Phys. Rev. B 69, 035111 (2004).

[3] I. Paul, C. Pepin, and M. R. Norman, Phys. Rev. Lett. 98, 026402 (2007) ; I. Paul, C. Pepin, M. R. Norman, Phys. Rev. B 78, 035109 (2008).

[4] C. Pepin, Phys. Rev. Lett. 98, 206401 (2007) ; C. Pepin, Phys. Rev. B 77, 245129 (2008).

[5] T. Moriya and J. Kawabata, J. Phys. Soc. Jpn. 34, 639 (1973) ; T. Moriya and J. Kawabata, J. Phys. Soc. Jpn. 35, 669 (1973) ; J. A. Hertz, Phys. Rev. B 14, 1165 (1976) ; A. J. Millis, Phys. Rev. B 48, 7183 (1993).

[6] A. Rosch, A. Schröder, O. Stockert, and H. v. Löhneysen, Phys. Rev. Lett. 79, 159 (1997).

[7] K. Miyake and H. Kohno, J. Phys. Soc. Jpn. 74, 254 (2005).

[8] K. Behnia, D. Jaccard, and J. Flouquet, J. Phys. : Condens. Matter 16, 5187 (2004).

[9] J. Benz et al., Physica B 259-261, 380 (1999).

[10] T. Kuwai et al., Physica B 378-380, 146 (2006).

[11] K. Izawa et al., Phys. Rev. Lett. 99, 147005 (2007).

[12] Z. Zhu et al., cond-mat/0907.0967 (unpublished).

[13] S. Hartmann, N. Oeschler, C. Krellner, C. Geibel and F. Steglich, Phys. Rev. Lett. 104, 096401 (2010).

[14] C.Grenzebach, F. B. Anders, G. Czycholl, and T.Pruschke, Phys. Rev. B 74, 195119 (2006).

[15] V. Zlatic̀, R. Monnier, and J. K. Freericks, Phys. Rev. B 78, 045113 (2008).

[16] I. Paul and G. Kotliar, Phys. Rev. B 64, 184414 (2001).

[17] Q. Si, S. Rabello, K. Ingersent, and L. Smith, Nature (London) 413, 804 (2001).

[18] L. B. Ioffe and G. Kotliar, Phys. Rev. B 42, 10348 (1990); P. A. Lee and N. Nagaosa, Phys. Rev. B 46, 5621 (1992).

[19] G. D. Mahan, Many-Particle Physics 3th ed. (Kluwer Academic/Plenum Publishers, New York, 2000).

[20] This half bandwidth is rather smaller than the estimated one [S. Friedemann et al., Phys. Rev. B 82, 035103 (2010)]. Since the Seebeck coefficient depends on the band structure heavily which is poorly described within the Kondo breakdown model, we introduce a phenomenological fitting parameter $A$ for describing the quantitative dispersion effect.

[21] Ki-Seok Kim and C. Pépin, Phys. Rev. B 81, 205108 (2010).

[22] K.-S. Kim, A. Benlagra, and C. Pépin, Phys. Rev. Lett. 101, 246403 (2008). 
[23] K.-S. Kim and C. Pépin, Phys. Rev. Lett. 102, 156404 (2009).

[24] S. Paschen et al., Nature 432, 881 (2004).
[25] S. Friedemann et al., PNAS 107, 14547 (2010).

[26] M.R. Norman, Phys. Rev. B 71, 220405(R), (2005). 